\title{
A Modal Multifield Approach for an Extended Flexible Body Description in Multibody Dynamics
}

\author{
ANDREAS HECKMANN ${ }^{1}$, MARTIN ARNOLD ${ }^{2}$ and ONDŘEJ VACULÍN ${ }^{1}$ \\ ${ }^{1}$ Institute of Robotics and Mechatronics, Vehicle System Dynamics, DLR German Aerospace Center, \\ P.O. Box 1116, 82230 Wessling, Germany \\ ${ }^{2}$ Institute of Numerical Mathematics, Department of Mathematics and Computer Science, \\ Martin-Luther-University Halle-Wittenberg, 06099 Halle (Saale), Germany; \\ E-mail: andreas.heckmann@DLR.de,URL:www.robotic.dlr.delfsd
}

(Received: 21 July 2003; accepted in revised form: 26 April 2004)

\begin{abstract}
This paper presents a new methodology to simulate the behaviour of flexible bodies influenced by multiple physical field quantities in addition to the classical mechanical terms. The theoretical framework is based on the extended Hamilton Principle and an adapted modal multifield approach. Furthermore, the use of finite element analysis for the necessary data preprocessing is explained. Numerical solution strategies for the coupled system of differential equations with different time scale properties are mentioned. The method is applied to simulate a structure with distributed piezo-ceramic devices inducing an additional electrostatic field. Two thermoelastic problems, which have to consider the influence of spatial temperature distribution, also demonstrate the benefits of the presented approach.
\end{abstract}

Keywords: modal multifield representation, thermoelasticity, piezoelectricity, flexible multibody systems

\section{Introduction}

Multibody dynamics focuses on the more global behaviour of mechanical systems. As a result the modelling task is simplified because in principle only a moderately detailed modelling level is required compared to the finite element approach. However, this attitude makes it necessary to handle all engineering disciplines and problems which significantly influence the technical system under evaluation.

In addition, the complexity of technical systems tends to increase involving more and more technical domains. Thus, multibody dynamics is exposed as a continuous work field. Important issues in this context are supplementary modelling capabilities and interfaces to other computational engineering tools [1].

Besides this general background, the extended representation of flexible bodies that will be introduced in the present paper is motivated by two specific fields of application. 
The first application example deals with so-called smart or adaptive structures. This concept has been developed to overcome the drawback of lightweight structures, viz. their susceptibility to vibrations. In vehicle applications it is aimed to achieve comfort improvements by the adaptive modification of the structure's response to various stimuli.

Thin piezo-ceramic patches, integrated into the structure, are one promising way to achieve this purpose. As a result an additional electrostatic field is induced by these piezo-ceramic actuators or sensors, which has to be considered for evaluating the behaviour of the flexible body.

Since smart structures are mechatronic devices, their design involves several engineering disciplines such as structural mechanics, electronics and control. The optimisation of such a complex system is a challenging task which may be supported advantageously by multibody dynamics as a system dynamics method.

The second application field refers to a classical problem of continuum mechanics, namely thermoelasticity. Usually, thermal expansion may be neglected in multibody dynamics, because the deformation of flexible bodies caused by temperature fields is small compared to displacements caused by mechanical forces. But such scenarios exist in which the consideration of both temperature and displacement field makes sense or may even be mandatory.

The specific importance of a combined thermal and elastic analysis can be stated for problems with large membrane or normal stresses due to temperature distribution. As a consequence, the natural frequencies of flexural vibrations and the related stiffness terms will decrease or even drop down to zero so that thermal buckling may occur.

A strong coupling between displacement and thermal field such as transient contact problems with heat generating friction, e.g. between brake disc and pad, also requires both thermal and elastic analysis.

The examples given in this paper were chosen to give an overview of these application fields. They use moderately complex single body models to enable a clear demonstration of the modelling particularities concerning thermoelasticity on the one hand and those regarding adaptive structures on the other. The lowdimensional representation of multiphysical, co-existent field quantities, achieved by the geometric semi-discretisation with just a few global modes, is a powerful novel approach to extend the simulation techniques of classical multibody dynamics to a new class of problems.

The present exposition covers the issues theory, data provision and verification. The application of the method to complex problems still remains an elaborate task involving e.g. the definition of reasonable boundary conditions, see [2] for a comprehensive simulation set up of industrial high-precision tooling machinery with thermally-induced tool center point displacements. 
Table I. Glossary of standard quantities and symbols.

\begin{tabular}{|c|c|c|c|}
\hline \multicolumn{4}{|c|}{ Basic quantities } \\
\hline$t$ & time & $m$ & mass \\
\hline$V$ & volume & $\varrho$ & density \\
\hline$B$ & boundary area & $\mathbf{n}_{B}$ & outer unit normal vector \\
\hline \multicolumn{4}{|c|}{ Mechanical quantities } \\
\hline $\mathbf{r}$ & position vector & $\mathbf{u}$ & displacement vector \\
\hline $\mathbf{v}$ & velocity & $\boldsymbol{\omega}$ & angular velocity \\
\hline$E$ & Young's module & $\varepsilon$ & strain tensor in vector format \\
\hline $\mathbf{x}$ & Lagrange co-ordinate & $\mathbf{f}$ & external force \\
\hline $\mathbf{a}$ & acceleration & $\alpha$ & angular acceleration \\
\hline$v$ & Poisson coefficient & $\sigma$ & stress tensor in vector format \\
\hline \multicolumn{4}{|c|}{ Thermal quantities } \\
\hline$\Theta$ & absolute temperature & $\mathbf{q}$ & heat flux \\
\hline$\eta$ & entropy density & $c$ & specific heat coefficient \\
\hline$\Lambda$ & thermal conductivity matrix & $\alpha$ & thermal expansion coefficient \\
\hline$S$ & heat source density & & \\
\hline \multicolumn{4}{|c|}{ Electrostatic quantities } \\
\hline$\varphi$ & electric potential & $\mathbf{e}$ & electric field strength \\
\hline d & electric displacement & $Q_{\varphi}$ & applied electric charge \\
\hline \multicolumn{4}{|c|}{ Indices and operators } \\
\hline()$_{V}$ & \multirow{4}{*}{\multicolumn{3}{|c|}{$\begin{array}{l}\text { The index } V \text { specifies a physical quantity as defined per volume, e.g. } \mathbf{f}_{V} \text { denotes a volume } \\
\text { The index } B \text { relates a quantity to the boundary surface, e.g. } \mathbf{f}_{B} \text { symbolises a surface load } \\
\text { The index } R \text { indicates motion terms of the flexible body's reference frame, see Section } 2.4 \\
\text { The tilde operator defines a vector matrix transformation used to replace the vector cross } \\
\text { product by a matrix multiplication: } \boldsymbol{a} \times \boldsymbol{b}=\tilde{\boldsymbol{a}} \boldsymbol{b}=-\tilde{\boldsymbol{b}} \boldsymbol{a}\end{array}$}} \\
\hline()$_{B}$ & & & \\
\hline()$_{R}$ & & & \\
\hline$(\sim)$ & & & \\
\hline
\end{tabular}

\section{Theoretical Framework}

\subsection{NOTATIONS AND DEFINITIONS}

The multifield description provides the governing equations for the mechanical, thermal and electrostatic fields. As a result, there is a whole bundle of variables in this section. The standard quantities are summarised in Table I.

\subsection{MATERIAL CONSTITUTION}

This paper deals with three physical fields, each specified by a pair of field variable terms. The mechanical state of a material particle is quantified by its stress and its strain tensor, the electrical state by its electric field strength and the electric displacement and the thermal state by its temperature and entropy density.

In order to describe the properties and influence of the material, a constitutive relation has to be found to quantify the thermodynamical state of a material point uniquely. 
If strain $\varepsilon$, electric field strength $\mathbf{e}$ and temperature $\Theta$ are chosen as independent variables, the electric Gibbs potential arises as associate function [3, Chapter 5]:

$$
\mathrm{d} G=-\varepsilon^{\mathrm{T}} \mathrm{d} \sigma-\mathbf{d}^{\mathrm{T}} \mathrm{d} \mathbf{e}-\eta \mathrm{d} \Theta .
$$

In praxis the introduction of a new variable $\vartheta$, replacing the absolute temperature $\Theta$ by the increment w.r.t. linearisation temperature $\Theta_{0}$ proved to be advantageous:

$$
\vartheta=\Theta-\Theta_{0} .
$$

The electric Gibbs potential, approximated by its second-order Taylor expansion at a natural state, in which $\vartheta$, $\mathbf{e}$ and $\varepsilon$ vanish, enables the formulation of a linear constitutive equation in matrix form: ${ }^{1}$

$$
\left(\begin{array}{l}
\boldsymbol{\sigma} \\
\mathbf{d} \\
\eta
\end{array}\right)=\left(\begin{array}{rrr}
\mathbf{H}_{c} & -\mathbf{H}_{e}^{\mathrm{T}} & -\mathbf{H}_{\lambda}^{\mathrm{T}} \\
\mathbf{H}_{e} & \mathbf{H}_{\epsilon} & \mathbf{H}_{p} \\
\mathbf{H}_{\lambda} & \mathbf{H}_{p}^{\mathrm{T}} & \mathbf{H}_{a}
\end{array}\right)\left(\begin{array}{l}
\varepsilon \\
\mathbf{e} \\
\vartheta
\end{array}\right)=\mathbf{H}\left(\begin{array}{l}
\varepsilon \\
\mathbf{e} \\
\vartheta
\end{array}\right) .
$$

The main diagonal elements of $\mathbf{H}$ specify the material properties of the monodisciplinary effects. $\mathbf{H}_{c}$ can be identified as the classical $6 \times 6$ elasticity tensor relating stress to strain, $\mathbf{H}_{\epsilon}$ consists of the permittivity coefficients and $\mathbf{H}_{a}=\varrho c / \Theta_{0}$ is the heat capacity coefficient, which relates temperature and entropy density.

In the un-coupled, isotropic thermoelastic problem the first row of (3) may be rewritten to extract the widely used thermal strain $\varepsilon_{\vartheta}[5$, Vol. 1, (4.26)]:

$$
\boldsymbol{\sigma}=\mathbf{H}_{c}\left(\varepsilon-\varepsilon_{\vartheta}\right) \quad \text { with } \quad \varepsilon_{\vartheta}=\mathbf{H}_{c}^{-1} \mathbf{H}_{\lambda}^{\mathrm{T}} \vartheta=\left(\begin{array}{llllll}
\alpha & \alpha & \alpha & 0 & 0 & 0
\end{array}\right)^{\mathrm{T}} \vartheta
$$

\subsection{GENERALISED HAMILTON'S PRINCIPLE}

Parkus [6] established the generalised Hamilton's Principle for coupled thermoelasticity, which was augmented for piezo-thermoelasticity by Nowacki [7]:

$$
\delta \int_{t_{1}}^{t_{2}}(T-\Pi+A) \mathrm{d} t=0, \quad \delta \int_{t_{1}}^{t_{2}} \Xi \mathrm{d} t=0 .
$$

The integrals in (4), which are stated to become stationary, use the following definitions:

$$
\begin{array}{ll}
T=\frac{1}{2} \int_{m} \dot{\mathbf{r}}^{\mathrm{T}} \dot{\mathbf{r}} \mathrm{d} m, & A=\int_{V} \mathbf{f}_{V}^{\mathrm{T}} \mathbf{r} \mathrm{d} V+\oint_{B}\left(\mathbf{f}_{B}^{\mathrm{T}} \mathbf{r}-\varphi Q_{\varphi}\right) \mathrm{d} B, \\
\Pi=\int_{V}(G+\eta \Theta) \mathrm{d} V, & \Xi=\int_{V}(H-\eta \Theta \Theta-S \Theta) \mathrm{d} V+\oint_{B} \mathbf{q}_{B}^{\mathrm{T}} \mathbf{n}_{B} \Theta \mathrm{d} B .
\end{array}
$$

\footnotetext{
${ }^{1}$ The indices of the material coefficient matrices are chosen analogously to [4, Chapter 24].
} 
In particular, the scalar potential $H$, called heat flux potential per volume, has to be pointed out because of its close relation to the fundamental Fourier law of heat conduction:

$$
H=\frac{1}{2}(\nabla \Theta)^{\mathrm{T}} \boldsymbol{\Lambda}(\nabla \Theta), \quad \Longrightarrow \quad \mathbf{q}=-\frac{\partial H}{\partial(\nabla \Theta)^{\mathrm{T}}}=-\boldsymbol{\Lambda}(\nabla \Theta) .
$$

In (4), only the independent field variables $\mathbf{r}$, e and $\Theta$ are varied while the other quantities are kept unchanged. This procedure is only admissible if all external quantities like $\mathbf{f}_{B}$ or $\mathbf{q}_{B}$ are monogenetic, i.e. could be derived out of a scalar function. They do not necessarily need to be conservative [8, Chapter 1].

Separation of variations for the three fields, substitution of $G$ in (4) using (1) and the fundamental lemma of the variational approach yield the field equations in weak form:

$$
\begin{array}{r}
\int_{V}\left[-\varrho \delta \mathbf{r}^{\mathrm{T}} \ddot{\mathbf{r}}-\boldsymbol{\sigma}^{\mathrm{T}} \delta \varepsilon+\mathbf{f}_{V}^{\mathrm{T}} \delta \mathbf{r}\right] \mathrm{d} V+\oint_{B} \mathbf{f}_{B}^{\mathrm{T}} \delta \mathbf{r} \mathrm{d} B=0, \\
\int_{V} \mathbf{d}^{\mathrm{T}} \delta \mathbf{e} \mathrm{d} V-\oint_{B} \delta \varphi Q_{\varphi} \mathrm{d} B=0, \\
\int_{V}\left[-(\nabla \delta \Theta)^{\mathrm{T}} \mathbf{q}+(\Theta \dot{\eta}-S) \delta \Theta\right] \mathrm{d} V+\oint_{B} \mathbf{q}_{B}^{\mathrm{T}} \mathbf{n}_{B} \delta \Theta \mathrm{d} B=0 .
\end{array}
$$

At first sight, the equations (6)-(8) look like three un-coupled field descriptions from mono-disciplinary engineering textbooks. But the coupling becomes obvious by eliminating the dependent field variables using (3).

\subsection{MODAL MULTIFIELD APPROACH}

The kinematics bases on a floating frame of reference formulation [9, Chapter 1] and thus gets the form:

$$
\begin{aligned}
& \mathbf{r}=\mathbf{r}_{R}+\mathbf{x}+\mathbf{u}, \\
& \dot{\mathbf{r}}=\mathbf{v}_{R}+\tilde{\boldsymbol{\omega}}_{R}(\mathbf{x}+\mathbf{u})+\dot{\mathbf{u}} \\
& \ddot{\mathbf{r}}=\mathbf{a}_{R}+\left(\tilde{\boldsymbol{\alpha}}_{R}+\tilde{\boldsymbol{\omega}}_{R} \tilde{\boldsymbol{\omega}}_{R}\right)(\mathbf{x}+\mathbf{u})+2 \tilde{\boldsymbol{\omega}}_{R} \dot{\mathbf{u}}+\ddot{\mathbf{u}} .
\end{aligned}
$$

The displacement of a body particle within the reference frame $\mathbf{u}=\mathbf{u}(\mathbf{x}, t)$ will be described with separated variables as the product of time-independent modal functions $\boldsymbol{\Phi}_{u}(\mathbf{x})$ by coefficients $\mathbf{z}_{u}(t)$. Within this approximation, the evaluation of the strain field is feasible by means of the differential operator $\mathbf{D}_{\epsilon u}$ [5, Vol. 1, (6.9)]:

$$
\mathbf{u}=\boldsymbol{\Phi}_{u} \mathbf{z}_{u}, \quad \varepsilon=\mathbf{D}_{\epsilon u} \mathbf{u}=\left(\mathbf{D}_{\epsilon u} \boldsymbol{\Phi}_{u}\right) \mathbf{z}_{u}=\mathbf{B}_{u} \mathbf{z}_{u}
$$


Regarding the electrostatics, the trial functions $\boldsymbol{\Phi}_{\varphi}$ describe the electrical potential field. Thus, the electrical field strength e results from a negative gradient operation:

$$
\varphi=\Phi_{\varphi} \mathbf{z}_{\varphi}, \quad \mathbf{e}=-\nabla \varphi=\left(-\nabla \boldsymbol{\Phi}_{\varphi}\right) \mathbf{z}_{\varphi}=\mathbf{B}_{\varphi} \mathbf{z}_{\varphi} .
$$

The analogous approach is chosen for the scalar temperature field. Here, the application of the $\nabla$ operator is necessary to evaluate the heat flux vector $\mathbf{q}$ :

$$
\vartheta=\boldsymbol{\Phi}_{\vartheta} \mathbf{z}_{\vartheta}, \quad \nabla \vartheta=\left(\nabla \boldsymbol{\Phi}_{\vartheta}\right) \mathbf{z}_{\vartheta}=\mathbf{B}_{\vartheta} \mathbf{z}_{\vartheta}, \quad \Longrightarrow \quad \mathbf{q}=-\boldsymbol{\Lambda} \mathbf{B}_{\vartheta} \mathbf{z}_{\vartheta} .
$$

\subsection{EQUATIONS OF MOTION}

Now the matrices $\mathbf{K}_{u u}, \mathbf{K}_{u \varphi}$ and $\mathbf{K}_{u \vartheta}$ are introduced for volume-dependent integrals which can be preprocessed and accessed during the time integration of the multibody system:

$$
\begin{aligned}
\mathbf{K}_{u u} & :=\int_{V} \mathbf{B}_{u}^{\mathrm{T}} \mathbf{H}_{c} \mathbf{B}_{u} \mathrm{~d} V, \\
\mathbf{K}_{u \varphi} & :=\int_{V} \mathbf{B}_{u}^{\mathrm{T}} \mathbf{H}_{e}^{\mathrm{T}} \mathbf{B}_{\varphi} \mathrm{d} V, \\
\mathbf{K}_{u \vartheta} & :=\int_{V} \mathbf{B}_{u}^{\mathrm{T}} \mathbf{H}_{\lambda}^{\mathrm{T}} \boldsymbol{\Phi}_{\vartheta} \mathrm{d} V .
\end{aligned}
$$

From the mechanical point of view, the thermal and the electrostatic field generate internal, distributed mechanical loads. Obviously, there is no direct influence on the inertia properties of the body. That is why the mass, gyroscopic and centripetal terms within the equations of motion can be adopted from literature. Shabana in [10, (5.140)] and Schwertassek and Wallrapp in [9, (6.308)] specified the generalised Newton-Euler equations for the unconstrained motion of a deformable body that undergoes large reference displacements.

A comparison of (6) with these references yields the extended equations of motion:

$$
\left(\begin{array}{ccc}
\mathbf{M}_{a a} & \mathbf{M}_{a \alpha} & \mathbf{M}_{a u} \\
& \mathbf{M}_{\alpha \alpha} & \mathbf{M}_{\alpha u} \\
\text { sym. } & & \mathbf{M}_{u u}
\end{array}\right)\left(\begin{array}{c}
\mathbf{a}_{R} \\
\boldsymbol{\alpha}_{R} \\
\ddot{\mathbf{z}}_{u}
\end{array}\right)=\left(\begin{array}{c}
\mathbf{h}_{a} \\
\mathbf{h}_{\alpha} \\
\mathbf{h}_{u}
\end{array}\right)+\left(\begin{array}{c}
0 \\
0 \\
-\mathbf{K}_{u u} \mathbf{z}_{u}+\mathbf{K}_{u \varphi} \mathbf{z}_{\varphi}+\mathbf{K}_{u \vartheta} \mathbf{z}_{\vartheta}
\end{array}\right)
$$

The mass matrix on the left-hand side of (14) is formulated as $3 \times 3$ block matrix such that the sub-matrices specify the inertia coupling between acceleration terms due to translational, angular and elastic motion, denoted by ()$_{a},()_{\alpha}$ and ( ) $)_{u}$. The right-hand side terms $\mathbf{h}_{a}, \mathbf{h}_{\alpha}$ and $\mathbf{h}_{u}$ summarise all inertia, damping and external forces. 
The added products $\mathbf{K}_{u \varphi} \mathbf{z}_{\varphi}$ and $\mathbf{K}_{u \vartheta} \mathbf{z}_{\vartheta}$ represent the influence of the electrostatic and the thermal field respectively on the equations of motion. They may be interpreted as modal forces acting on the elastic body.

Although the thermal and electrostatic loads do not alter the inertia quantities in (14), the displacements caused by these loads do, since the mass matrix and the vectors $\mathbf{h}_{a}$ and $\mathbf{h}_{\alpha}$ depend on the deformation state of the body.

\subsection{ELECTROSTATIC EQUATION}

With

$$
\begin{aligned}
\mathbf{K}_{\varphi \varphi} & :=\int_{V} \mathbf{B}_{\varphi}^{\mathrm{T}} \mathbf{H}_{\epsilon} \mathbf{B}_{\varphi} \mathrm{d} V, \\
\mathbf{K}_{\varphi \vartheta} & :=\int_{V} \mathbf{B}_{\varphi}^{\mathrm{T}} \mathbf{H}_{p} \boldsymbol{\Phi}_{\vartheta} \mathrm{d} V, \\
\mathbf{Q}_{\varphi} & :=\oint_{B} \boldsymbol{\Phi}_{\varphi}^{\mathrm{T}} Q_{\varphi} \mathrm{d} B .
\end{aligned}
$$

Equation (7) can be rewritten:

$$
\mathbf{Q}_{\varphi}=\mathbf{K}_{\varphi \varphi} \mathbf{z}_{\varphi}+\mathbf{K}_{u \varphi}^{\mathrm{T}} \mathbf{z}_{u}+\mathbf{K}_{\varphi \vartheta} \mathbf{z}_{\vartheta}
$$

The algebraic sensor equation (16) is needed to calculate the electric quantities, e.g. the electric charges $\mathbf{Q}_{\varphi}$, if the piezo-ceramic components are used as sensors or, more generally, if they are part of arbitrary electric circuits, see [11, Chapter 3] and [12]. Analogously to (14), the terms $\mathbf{K}_{\varphi \vartheta}$ and $\mathbf{K}_{u \varphi}^{\mathrm{T}}=\mathbf{K}_{\varphi u}$ represent coupling matrices, which transform the thermal and the displacement quantities into the electrostatic field equation.

\subsection{THERMAL EQUATION}

In (8), the natural boundary conditions are represented by the heat flux through the boundary surface. It depends on the physical circumstances how this term has to be introduced into the thermal equation. For Neumann conditions, the boundary heat flux $q_{\mathrm{B}}$ is given explicitly. If convection occurs on the boundary surface, a Robin or mixed boundary condition is imposed, specified by the film coefficient $h_{\mathrm{f}}$ and the bulk temperature $\vartheta_{\infty}$ of the fluid [13, Section 4.1]. Although this list is not complete, we confine ourselves to these two cases:

$$
\mathbf{q}_{\mathrm{B}}^{\mathrm{T}} \mathbf{n}_{\mathrm{B}}=-q_{\mathrm{B}}-h_{\mathrm{f}}\left(\vartheta_{\mathrm{B}}-\vartheta_{\infty}\right)
$$

Besides the thermal-mechanical coupling matrix $\mathbf{C}_{\vartheta u}=\Theta_{0} \mathbf{K}_{u \vartheta}^{\mathrm{T}}$ and the thermalelectrostatic coupling term $\mathbf{C}_{\vartheta \varphi}=\Theta_{0} \mathbf{K}_{\varphi \vartheta}^{\mathrm{T}}$, which may be derived from their 
corresponding transposed quantities, the following notations are used for geometric integrals:

$$
\begin{aligned}
\mathbf{C}_{\vartheta \vartheta}:=\int_{V} \Theta_{o} \boldsymbol{\Phi}_{\vartheta}^{\mathrm{T}} \mathbf{H}_{a} \boldsymbol{\Phi}_{\vartheta} \mathrm{d} V, & \mathbf{K}_{\vartheta R}:=\oint_{B} h_{\mathrm{f}} \boldsymbol{\Phi}_{\vartheta}^{\mathrm{T}} \boldsymbol{\Phi}_{\vartheta} \mathrm{d} B, \\
\mathbf{K}_{\vartheta \vartheta}:=\int_{V} \mathbf{B}_{\vartheta}^{\mathrm{T}} \boldsymbol{\Lambda} \mathbf{B}_{\vartheta} \mathrm{d} V, & \mathbf{Q}_{\vartheta R}:=\oint_{B} \boldsymbol{\Phi}_{\vartheta}^{\mathrm{T}} h_{\mathrm{f}} \mathrm{d} B, \\
\mathbf{Q}_{\vartheta S}:=\int_{V} \boldsymbol{\Phi}_{\vartheta}^{\mathrm{T}} \mathrm{d} V, & \mathbf{Q}_{\vartheta N}:=\oint_{B} \boldsymbol{\Phi}_{\vartheta}^{\mathrm{T}} \mathrm{d} B .
\end{aligned}
$$

Finally, the coupled, linearised thermal equation can be stated:

$$
\begin{aligned}
& \mathbf{C}_{\vartheta \vartheta} \dot{\mathbf{z}}_{\vartheta}+\mathbf{C}_{\vartheta \varphi} \dot{\mathbf{z}}_{\varphi}+\mathbf{C}_{\vartheta u} \dot{\mathbf{z}}_{u}+\left(\mathbf{K}_{\vartheta \vartheta}+\mathbf{K}_{\vartheta R}\right) \mathbf{z}_{\vartheta} \\
& =\mathbf{Q}_{\vartheta S} S_{u}+\mathbf{Q}_{\vartheta N} q_{B}+\mathbf{Q}_{\vartheta R} \vartheta_{\infty} .
\end{aligned}
$$

The generalised velocities $\dot{\mathbf{z}}_{u}$ in (19) indicate that the temperature field depends on the displacements and the strains. Whereas the thermal effect on the displacements is well known and widely accounted for in finite element analysis, the retroaction from displacements on temperatures, called the Gough-Joule effect [14], is very frequently neglected because of its limited influence on the temperatures compared to the other terms.

If it is intended to identify the well-known un-coupled heat conduction equation of solids, (19) can be rewritten assuming $\mathbf{C}_{\vartheta u} \approx \mathbf{0}$ and $\mathbf{C}_{\vartheta \varphi} \approx \mathbf{0}$, cf. [5, Vol. 1, Section 17.2].

\subsection{TOPOLOGICAL ASPECTS}

Equations (14), (16) and (19) are to be posted for each body of the articulated mechanism under consideration. For a global representation (14) and (19) are rewritten in condensed form for a general elastic body ()$^{(i)}$ with electrostatic and thermal properties:

$$
\begin{aligned}
\mathbf{M}^{(i)} \ddot{\mathbf{z}}^{(i)} & =\mathbf{h}_{o}^{(i)}+\mathbf{h}_{m}^{(i)}\left(\mathbf{z}_{\varphi}^{(i)}, \mathbf{z}_{\vartheta}^{(i)}, \mathbf{z}_{u}^{(i)}\right), \\
\dot{\mathbf{z}}_{\vartheta}^{(i)} & =\mathbf{c}^{(i)}\left(\dot{\mathbf{z}}_{\varphi}^{(i)}, \mathbf{z}_{\vartheta}^{(i)}, \dot{\mathbf{z}}_{u}^{(i)}\right) .
\end{aligned}
$$

Besides the mechanical description (20) the set up of a piezo-thermoelastic body requires the definition of two additional, uniquely assigned elements. The thermal element reflects (21) and evaluates the thermal state of the body.

The electrostatic element stands for the measurement capabilties of the piezoceramic devices attached to body ( $)^{(i)}$ and calculates the electric charges $\mathbf{Q}_{\varphi}^{(i)}$, i.e. 
the sensor output of the piezo-patches according to (16):

$$
\mathbf{Q}_{\varphi}^{(i)}=\mathbf{d}^{(i)}\left(\mathbf{z}_{\varphi}^{(i)}, \mathbf{z}_{\vartheta}^{(i)}, \mathbf{z}_{u}^{(i)}\right)
$$

The actuation capabilties of the piezo-patches are reflected by the input variable $\mathbf{z}_{\varphi}^{(i)}$ in (20). From that point of view the elastic body ( $)^{(i)}$ may be interpreted as a controlled plant, whereas $\mathbf{z}_{\varphi}^{(i)}$ represents its input and $\mathbf{Q}_{\varphi}^{(i)}$ its output. These quantities are supposed to be used for the set-up of an appropriate control law such as $\mathbf{z}_{\varphi}^{(i)}=\mathbf{z}_{\varphi}^{(i)}\left(\mathbf{Q}_{\varphi}^{(i)}\right)$, see the piezoelectric application in Section 4.

Mechanical interactions between separated bodies of a mechanism are to be modelled either as applied forces or by kinematical constraints. However, (20)(22) presume that the electrostatic and thermal field of body ( $)^{(i)}$ do not interfere with those of other bodies.

The model equations of a general elastic body with electrostatic and thermal features have been implemented in a developer version of the industrial multibody code SIMPACK.

In SIMPACK, the use of relative joint co-ordinates p enables an efficient recursive assembly of the equations of motion for the complete multibody system by an explicit $\mathcal{O}(N)$-formalism [15].

For tree-like structures the relative co-ordinates $\mathbf{p}$ are defined as minimum set of generalised mechanical co-ordinates. The pure mechanical part of the equations of motion reads [16]:

$$
\sum_{(i)}\left[\frac{\mathbf{z}^{(i)}}{\mathbf{p}}\right]^{\mathrm{T}}\left[\mathbf{M}^{(i)} \ddot{\mathbf{z}}^{(i)}-\mathbf{h}_{o}^{(i)}-\mathbf{h}_{m}^{(i)}\right]=\overline{\mathbf{M}} \ddot{\mathbf{p}}-\overline{\mathbf{h}}=\mathbf{0} .
$$

$\overline{\mathbf{M}}(\mathbf{p}, t)$ represents the symmetric inertia matrix of the complete multibody system. The generalised Coriolis and applied forces together with the generalised loads due to thermal and electrostatic influences are included in $\overline{\mathbf{h}}\left(\dot{\mathbf{p}}, \mathbf{p}, \overline{\mathbf{z}}_{\vartheta}\right.$, $\left.\overline{\mathbf{z}}_{\varphi}, t\right)$.

For closed-loop systems, Equations (23) are extended by kinematical constraints and the associated passive forces, see [10, Section 5.9]. Additional differential state equations result from the thermal features, see (21).

In its most general form the model equations of the complete system are given by:

$$
\begin{aligned}
\overline{\mathbf{M}}(\mathbf{p}, t) \ddot{\mathbf{p}} & =\overline{\mathbf{h}}\left(\dot{\mathbf{p}}, \mathbf{p}, \overline{\mathbf{z}}_{\varphi}, \overline{\mathbf{z}}_{\vartheta}, t\right)-\overline{\mathbf{G}}^{\mathrm{T}}(\mathbf{p}, t) \boldsymbol{\lambda} \\
\dot{\mathbf{z}}_{\vartheta} & =\overline{\mathbf{c}}\left(\dot{\mathbf{p}}, \overline{\mathbf{z}}_{\varphi}, \overline{\mathbf{z}}_{\vartheta}\right), \\
\mathbf{0} & =\overline{\mathbf{g}}(\mathbf{p}, t)
\end{aligned}
$$

with the constraint matrix $\overline{\mathbf{G}}:=\left(\frac{\overline{\mathbf{g}}}{\mathbf{p}}\right)(\mathbf{p}, t)$. 


\section{Solution Methods}

\subsection{DATA PREPROCESSING}

\subsubsection{Electrostatic Field Data}

It is state-of-the-art of industrial multibody tools to incorporate the results of an appropriate finite element analysis to obtain the mechanical data of a flexible body.

This approach may not yet be carried over to the data of smart structures. Although the finite element modelling of piezoelectric devices on shell elements is a field of active research [17], it is not yet introduced in an industrial finite element tool. To enable nevertheless the simulation of lightweight structures with shell elements, the following technique uses only purely mechanical data which are readily available.

Imagine a finite element model with shell elements. A modal analysis yields discrete mode matrices for every node $k$, located at the position $\mathbf{x}_{k} \in R^{3}$ which specify the displacements $\boldsymbol{\Phi}_{u, k} \in R^{3, m}$ and rotations $\boldsymbol{\Psi}_{u, k} \in R^{3, m}$ as functions of all observed modes $j, 1 \leq j \leq m$. The aim is to obtain the matrices $\mathbf{K}_{u \varphi}$ and $\mathbf{K}_{\varphi \varphi}$ for a piezo-ceramic patch, located upon a shell element, defined geometrically by the four nodes $k=1, \ldots, 4$, one at each corner.

For interpolation the shell mid-plane is mapped on a normalised $(\xi, \zeta)$-area. The interpolation functions may be organised by defining a matrix $\mathbf{N} \in R^{3,12}$ :

$$
\begin{aligned}
\mathbf{N} & =\left(N_{1} \mathbf{I}_{3} N_{2} \mathbf{I}_{3} N_{2} \mathbf{I}_{3} N_{4} \mathbf{I}_{3}\right), \\
N_{1} & =\frac{1}{4}(1-\xi)(1-\zeta), \quad N_{2}=\frac{1}{4}(1+\xi)(1-\zeta), \quad \mathbf{I}_{3}=\operatorname{diag}\{1,1,1\}, \\
N_{3} & =\frac{1}{4}(1-\xi)(1+\zeta), \quad N_{4}=\frac{1}{4}(1+\xi)(1+\zeta), \quad-1 \leq \xi, \quad \zeta \leq 1 .
\end{aligned}
$$

Now an iso-parametric approximation of the geometry $\mathbf{x}_{s}$ and the displacements $\mathbf{u}_{s}$ of a shell point, specified by $\boldsymbol{\xi}=\left(\xi, \zeta, t_{s}\right)^{\mathrm{T}}$, can be formulated:

$$
\mathbf{x}_{s}(\boldsymbol{\xi})=\mathbf{N} \mathbf{x}^{e}+t_{s} \mathbf{N} \mathbf{n}^{e}, \quad \mathbf{u}_{s}=\boldsymbol{\Phi}_{u, s}(\boldsymbol{\xi}) \mathbf{z}_{u}=\left(\mathbf{N} \boldsymbol{\Phi}^{e}-t_{s} \mathbf{N} \boldsymbol{\Psi}^{e}\right) \mathbf{z}_{u}, \quad \boldsymbol{\Phi}_{u, s} \in R^{3, m} .
$$

Here, the vector $\mathbf{x}^{e}$ organises the four-node positions, $\mathbf{n}^{e}$ summarises the unit normals to the shell mid-plane in the node points $\mathbf{n}_{k}$ and the matrices $\boldsymbol{\Phi}^{e}$ and $\boldsymbol{\Psi}^{e}$ represent the pre-described node displacements and rotations:

$$
\boldsymbol{\Phi}^{e}=\left(\begin{array}{c}
\boldsymbol{\Phi}_{u, 1} \\
\boldsymbol{\Phi}_{u, 2} \\
\boldsymbol{\Phi}_{u, 3} \\
\boldsymbol{\Phi}_{u, 4}
\end{array}\right), \quad \boldsymbol{\Psi}^{e}=\left(\begin{array}{c}
\tilde{\mathbf{n}}_{1} \boldsymbol{\Psi}_{u, 1} \\
\tilde{\mathbf{n}}_{2} \boldsymbol{\Psi}_{u, 2} \\
\tilde{\mathbf{n}}_{3} \boldsymbol{\Psi}_{u, 3} \\
\tilde{\mathbf{n}}_{4} \boldsymbol{\Psi}_{u, 4}
\end{array}\right), \quad \mathbf{x}^{e}=\left(\begin{array}{c}
\mathbf{x}_{1} \\
\mathbf{x}_{2} \\
\mathbf{x}_{3} \\
\mathbf{x}_{4}
\end{array}\right), \quad \mathbf{n}^{e}=\left(\begin{array}{l}
\mathbf{n}_{1} \\
\mathbf{n}_{2} \\
\mathbf{n}_{3} \\
\mathbf{n}_{4}
\end{array}\right) .
$$


Using this approximation of the displacements in modal description, the strain field may be evaluated using a differential strain-displacement operator $\mathbf{D}_{\epsilon u}$, e.g. according the Reissner-Mindlin assumption [5, Vol. 2, Chapter 8].

If it is additionally assumed that the electric potential varies linearly between the two electrodes of a piezo-patch, the related volume integrals (15) are feasible for evaluation, see [18] for further details.

\subsubsection{Thermal Field Data}

The analysis of temperature fields and the application of thermal loads on mechanical structures are widely used operations of the finite element method. The strategy to describe here organises the access to existing finite element data and the transfer into the multibody representation. The four steps to be done are additionally visualised in Figure 1:

1. Firstly, the thermal finite element description has to be reduced. Therefore, the modal approach in (12) is rewritten in discretised form denoting the number of thermal degrees of freedom of the finite element system by $n_{\vartheta}$ and of the multibody system by $m_{\vartheta}$ :

$$
\boldsymbol{\vartheta}=\boldsymbol{\Phi}_{\vartheta} \mathbf{z}_{\vartheta} \quad \text { with } \quad \boldsymbol{\Phi}_{\vartheta}=\left[\cdots \mathbf{a}_{i} \cdots\right], \quad 1 \leq i \leq m_{\vartheta}, \quad \mathbf{a}_{i} \in R^{n_{\vartheta}} .
$$

Each vector $\mathbf{a}_{i}$ represents a discrete thermal mode, i.e. assigns one temperature to each finite element degree of freedom. A mode may be a solution of the

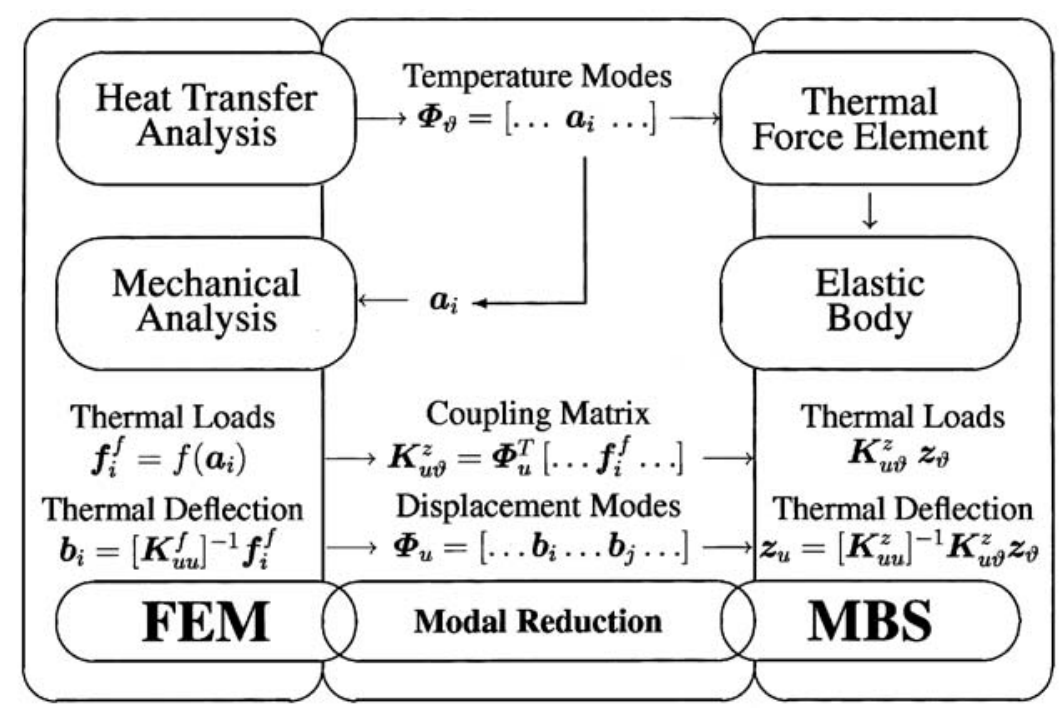

Figure 1. Thermal response modes (thermal deflections are assumed to be quasi-stationary). 
thermal eigenvalue problem $\left[\mathbf{C}_{\vartheta \vartheta}^{f} \kappa_{i}+\mathbf{K}_{\vartheta \vartheta}^{f}\right] \mathbf{a}_{i}=0$ or a solution of a frequency response or steady-state problem. ${ }^{2}$

2. The second step consists of a static analysis of the mechanical system. Each selected thermal mode $\mathbf{a}_{i}$ constitutes one mechanical load vector $\mathbf{f}_{i}^{f}$ and results in one corresponding displacement solution $\mathbf{b}_{i}$, further on called a thermal response mode:

$$
\mathbf{f}_{i}^{f}=\mathbf{f}_{i}^{f}\left(\mathbf{a}_{i}\right), \quad \mathbf{K}_{u u}^{f} \mathbf{b}_{i}=\mathbf{f}_{i}^{f}, \quad \mathbf{b}_{i} \in R^{n_{u}} .
$$

3. In the third step, additional displacement modes have to be evaluated and selected that represent the native mechanical behaviour of the system. See [19] for appropriate mode selection techniques. Equation (10) can then be rewritten in discretised form:

$$
\begin{aligned}
\mathbf{u} & =\boldsymbol{\Phi}_{u} \mathbf{z}_{u}, \\
\boldsymbol{\Phi}_{u} & =\left[\cdots \mathbf{b}_{i} \cdots \mathbf{b}_{j} \cdots\right], \quad m_{\vartheta}<j \leq m_{u}, \quad \mathbf{b}_{i}, \mathbf{b}_{j} \in R^{n_{u}} .
\end{aligned}
$$

If the column vectors of $\boldsymbol{\Phi}_{u}$ are linearly dependent, a maximum subset of linearly independent column vectors is selected to meet the demands of the Ritz approach. But the verification examples given later show that this situation is not likely to occur since both kinds of displacement solutions are of completely different nature.

4. Equations (27) and (29) enable a modal transformation of the thermal and the mechanical system out of their finite element formulation into the multibody description [9, Chapter 6]. The thermal-mechanical coupling matrix can be provided as the reorganisation of the thermal load vectors $\mathbf{f}_{i}^{f}$ :

$$
\mathbf{K}_{\vartheta u}^{z}=\boldsymbol{\Phi}_{u}^{\mathrm{T}} \mathbf{K}_{\vartheta u}^{f}, \quad \text { with } \quad \mathbf{K}_{\vartheta u}^{f}=\left[\cdots \mathbf{f}_{i}^{f} \cdots\right]
$$

Finally, all data needed to run electrostatic-mechanical and thermal-mechanical multibody simulations were made available.

\subsection{TIME INTEGRATION}

The numerical solvers for time integration benefit strongly from the modal reduction in the preprocessing step: modal reduction decreases the number of degrees of freedom by several orders of magnitude. Furthermore, the full finite element models have high-frequency solution components that are physically not relevant because of structural damping and damping in joints. Modal reduction

\footnotetext{
${ }^{2}$ The superscripts ( $)^{f}$ and ()$^{z}$ are used to distinguish corresponding terms in finite element and multibody representation, if they could be mixed up.
} 
eliminates these high-frequency components analytically and avoids in this way numerical instabilities that would result in strong restrictions of the time step size $\Delta_{t}$.

Today, the selection of trial functions $\boldsymbol{\Phi}_{u}, \boldsymbol{\Phi}_{\varphi}, \boldsymbol{\Phi}_{\vartheta}$ relies on the intuition of the engineer and on simplified linear considerations [19]. First attempts to select $\boldsymbol{\Phi}_{u}$, $\Phi_{\varphi}, \Phi_{\vartheta}$ adaptively use the truncation error in a fully nonlinear flexible multibody system model that is based on a formulation as saddle point problem in Sobolev spaces [20].

The model equations (24) form a second-order differential-algebraic equation (DAE). Classical time integration methods from computational mechanics like Newmark's scheme exploit this second-order structure explicitly. Industrial multibody system simulation packages do not follow this approach but transform (24) to an equivalent first-order system introducing the velocities $\mathbf{w}:=\dot{\mathbf{p}}$.

Following a proposal of Gear et al. [21], the DAE (24) is furthermore transformed to the analytically equivalent stabilised index-2 formulation (31) to avoid numerical instabilities in DAE time integration:

$$
\begin{aligned}
\dot{\mathbf{p}} & =\mathbf{w}-\overline{\mathbf{G}}^{\mathrm{T}}(\mathbf{p}, t) \boldsymbol{\eta}, \\
\overline{\mathbf{M}}(\mathbf{p}, t) \dot{\mathbf{w}} & =\overline{\mathbf{h}}\left(\mathbf{w}, \mathbf{p}, \overline{\mathbf{z}}_{\varphi}, \overline{\mathbf{z}}_{\vartheta}, t\right)-\overline{\mathbf{G}}^{\mathrm{T}}(\mathbf{p}, t) \boldsymbol{\lambda}, \\
\dot{\mathbf{z}}_{\vartheta} & =\overline{\mathbf{c}}\left(\mathbf{w}, \dot{\overline{\mathbf{z}}}_{\varphi}, \overline{\mathbf{z}}_{\vartheta}, t\right) \\
\mathbf{0} & =\overline{\mathbf{g}}(\mathbf{p}, t) \\
\mathbf{0} & =\overline{\mathbf{G}}(\mathbf{p}, t) \mathbf{w}(t)+\overline{\mathbf{g}}_{, t}(\mathbf{p}, t)
\end{aligned}
$$

This formulation makes explicit use of the constraints

$$
\mathbf{0}=\frac{\mathrm{d} \overline{\mathbf{g}}(\mathbf{p}, t)}{\mathrm{d} t}=\frac{\partial \overline{\mathbf{g}}}{\partial \mathbf{p}}(\mathbf{p}, t) \dot{\mathbf{p}}(t)+\frac{\partial \overline{\mathbf{g}}}{\partial t}(\mathbf{p}, t)=\overline{\mathbf{G}}(\mathbf{p}, t) \mathbf{w}(t)+\overline{\mathbf{g}}_{, t}(\mathbf{p}, t)
$$

on the level of velocity co-ordinates $\mathbf{w}$ and introduces an artificial correction term $\overline{\mathbf{G}}^{\mathrm{T}}(\mathbf{p}, t) \boldsymbol{\eta}$ with auxiliary variables $\boldsymbol{\eta}$ in the kinematical equations $\dot{\mathbf{p}}-\mathbf{w}=\mathbf{0}$. These variables $\boldsymbol{\eta}$ vanish identically for the analytical solution and remain in the size of the discretisation error during time integration.

The stabilised index-2 formulation (31) can be solved by any standard DAE time integration method like BDF or (implicit) Runge-Kutta methods. The industrial simulation packages MSC.ADAMS and SIMPACK offer adapted versions of the variable step-size variable order BDF-code DASSL [21] as default integrators.

These basic strategies for time integration in industrial multibody system simulation emphasize again that multibody system tools are suitable integration platforms for multiphysical problems. First-order differential equations like (19) and algebraic equations like (16) that describe the behaviour of non-mechanical system 
components may be added to the equations of motion (23) without any modifications of the BDF time integration method.

The standard time integration methods of multibody dynamics may even be applied if the modal approach involves a large number of trial functions, e.g. to resolve local effects in elastic structures. But in this special case co-simulation techniques combined with semi-analytical methods for the time integration of the modal equations proved to be substantially more efficient $[1,22,23]$.

\section{Piezoelectric Application}

A metal sheet equipped with piezo-elements to control the vibration is presented as an example to demonstrate the feasibility of the proposed methodology for modelling of piezo-elements in multibody systems. The description of piezo-elements has been implemented in the multibody simulation package SIMPACK [24].

\subsection{SIMULATION ENVIRONMENT}

The elastic structure of the metal sheet is modelled in ANSYS and transformed to its modal representation, which can be used as an elastic body representation for SIMPACK and which also serves as a base for controller design in MATLAB.

The metal sheet and the piezo-patches are modelled and simulated in SIMPACK. The controller design and simulation is performed in MATLAB/Simulink. SIM$P A C K$ and MATLAB/Simulink are connected via an inter-process communication interface [25].

\subsection{MODEL DESCRIPTION AND SIMULATION SCENARIO}

The model of an elastic metal sheet $\left(E=2.1 \times 10^{11} \mathrm{~Pa}, v=0.3, \varrho=7850 \mathrm{~kg} / \mathrm{m}^{3}\right)$ of size $1 \mathrm{~m} \times 1.3 \mathrm{~m}$ and $0.9 \mathrm{~mm}$ thin is studied. The displacements on the four corners are constrained to be zero. The model considers 14 eigenvalues ranging up to $20 \mathrm{~Hz}$. The structural damping is set to 0.01 . The piezo-elements, $0.4 \mathrm{~mm}$ thin $\left(H_{\epsilon, 33}=1.3 \times 10^{-8} \mathrm{~F} / \mathrm{m}, H_{e, 31}=-6.5 \mathrm{C} / \mathrm{m}^{2}\right)$, are attached on both sides of 140 finite elements visualised by the mesh in Figure 2. Such piezo-elements provide approximately linear behaviour up to the voltage of $400 \mathrm{~V}$. If higher voltage is applied, the piezo-elements behave nonlinearly and expose hysteresis effects.

Transformation of the model to a state space form is of advantage for the controller design:

$$
\begin{aligned}
\dot{\chi} & =\mathbf{A} \chi+\mathbf{B} v \\
\iota & =\mathbf{C} \chi+\mathbf{D} v
\end{aligned}
$$


where $\chi$ is the state, $\boldsymbol{v}$ the input and $\iota$ the output vector. $\mathbf{A}, \mathbf{B}, \mathbf{C}$ and $\mathbf{D}$ are the system matrices defined as follows:

$$
\begin{array}{ll}
\mathbf{A}=\left(\begin{array}{cc}
\mathbf{O} & \mathbf{I} \\
-\mathbf{M}_{u u}^{-1} \mathbf{K}_{u u} & -\mathbf{M}_{u u}^{-1} \mathbf{D}_{u u}
\end{array}\right), & \mathbf{C}=\left(\mathbf{K}_{u \varphi}^{\mathrm{T}} \mathbf{O}\right), \\
\mathbf{B}=\left(\begin{array}{c}
\mathbf{O} \\
-\mathbf{M}_{u u}^{-1} \mathbf{K}_{u \varphi}
\end{array}\right), & \mathbf{D}=\left(\mathbf{K}_{\varphi \varphi}^{\mathrm{T}}\right),
\end{array}
$$

where matrices $\mathbf{M}_{u u}, \mathbf{K}_{u u}, \mathbf{K}_{u \varphi}$ and $\mathbf{K}_{\varphi \varphi}$ are defined in (13), (14) and (15), matrix $\mathbf{D}_{u u}$ represents the structural damping, matrix $\mathbf{I}$ is the identity matrix and matrix $\mathbf{O}$ is the zero matrix.

The elastic metal sheet is excited at time $0.1 \mathrm{~s}$ with a force impact in the centre position. The force impact is characterised by the amplitude of $20 \mathrm{~N}$ and length $0.01 \mathrm{~s}$. The goal is to minimise the acceleration at the centre of the metal sheet.

\subsection{CONTROL DESIGN AND SELECTION OF PATCHES}

Traditional LQR control has been applied to design a controller for the metal sheet. The model has in its initial version 280 piezo-patches, which serve as actuators and as sensors, i.e. the system has 280 inputs and 280 outputs. Since the model contains 14 modes, the state space model is of 28 th order. The states are fully controllable and observable, but the output vector includes output voltage of piezo-patches instead of states, which are needed for the LQR design. However, one can construct a state estimate $\hat{\chi}$ such that the control law retains similar closed-loop properties [11].

The first step in the control design process is the selection of parameters of the weighting matrix $\mathbf{Q}$ in the $\mathrm{LQR}$ design cost function:

$$
J=\int_{0}^{\infty}\left(\chi^{\mathrm{T}} \mathbf{Q} \chi+\boldsymbol{v}^{\mathrm{T}} \mathbf{R} \boldsymbol{v}\right) \mathrm{d} t .
$$

The $\mathbf{Q}$ matrix has the block structure:

$$
\mathbf{Q}=k_{Q}\left(\begin{array}{cc}
\mathbf{Q}_{11} & \mathbf{O} \\
\mathbf{O} & \mathbf{O}
\end{array}\right)
$$

where $k_{Q}$ is a scalar parameter and $\mathbf{Q}_{11}$ is a diagonal matrix. The main diagonal entries of $\mathbf{Q}_{11}$ are the $z$ co-ordinates in matrix $\boldsymbol{\Phi}_{u, k}$ (see Section 3.1.1.) multiplied by the corresponding eigenfrequencies $\omega_{i}$.

In the beginning, it is necessary to identify the eigenmodes which are to be controlled, i.e. which have influence on the motion of the centre of the metal sheet. 


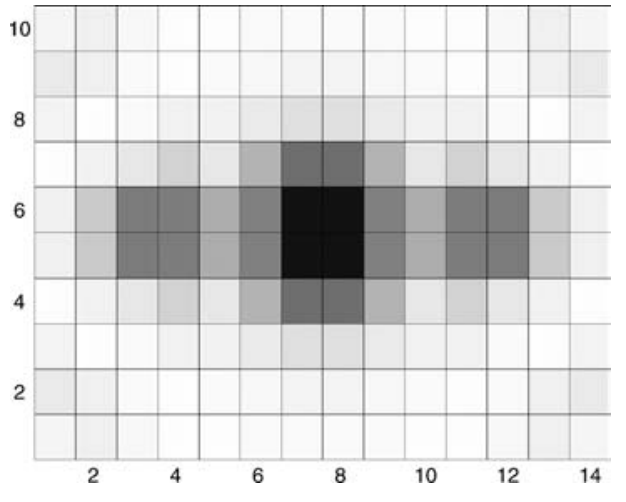

(Figure 2)

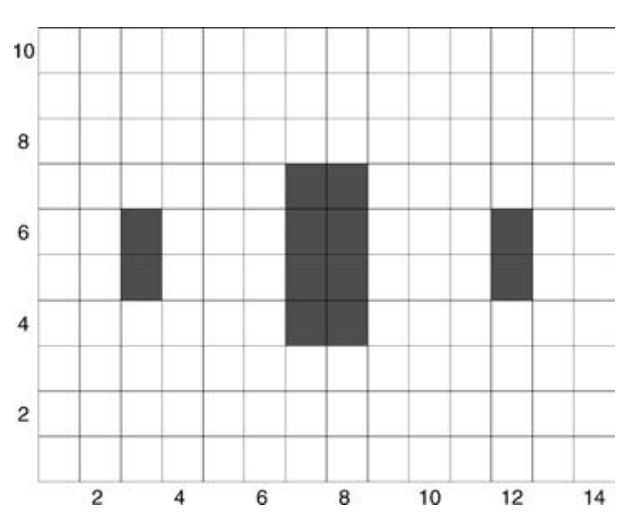

(Figure 3)

Figure 2. Mesh with piezo-patches, grey scaled according to their importance for control.

Figure 3. Patches selected for control.

According to the matrix $\boldsymbol{\Phi}_{u, k}$ the modes 1, 4, 7, 11 and 14 contribute to the motion in the $z$-direction. The other elements in $z$-direction of the matrix $\boldsymbol{\Phi}_{u, k}$ for the centre of the sheet are zero.

In the second step, the piezoelectric patches will be selected, which will be used for the controller of the metal sheet. The selection criterion directly bases on the feedback gain $\mathbf{K}$ of the LQR controller $\boldsymbol{v}=-\mathbf{K} \boldsymbol{\chi}$. The matrix $\mathbf{K}$ is an $r \times n$ matrix, where $r$ is the number of inputs and $n$ is the number of states of the controlled system. Since the inputs represent the piezo-patches, the most important patches should have the largest norm $\zeta_{i}$ of the corresponding column vector in the matrix $\mathbf{K}$, e.g.:

$$
\zeta_{i}=\left(\sum_{j=1}^{n}\left|k_{i, j}\right|^{2}\right)^{\frac{1}{2}}, \quad 1 \leq i \leq r .
$$

Since the system is symmetric w.r.t. two main axes and the patches are located on both sides of the metal sheet (collocated patches), the final number of patches will be a multiple of eight. The contribution of the patches to the control of the sheet's centre point is illustrated in Figure 2. According to the results presented in Figure 2, the most important patches are selected, see Figure 3. Because of feasibility, the final configuration has 24 patches, 12 most important patches on each side of the plate.

In the third step, after selection of the reduced set of patches, a new LQR design and observer design should be performed and the parameter $k_{Q}$ should be tuned in order to use the patches as efficiently as possible, i.e. the controller should use the whole linear range of the piezo-element. The Simulink block diagram of the 


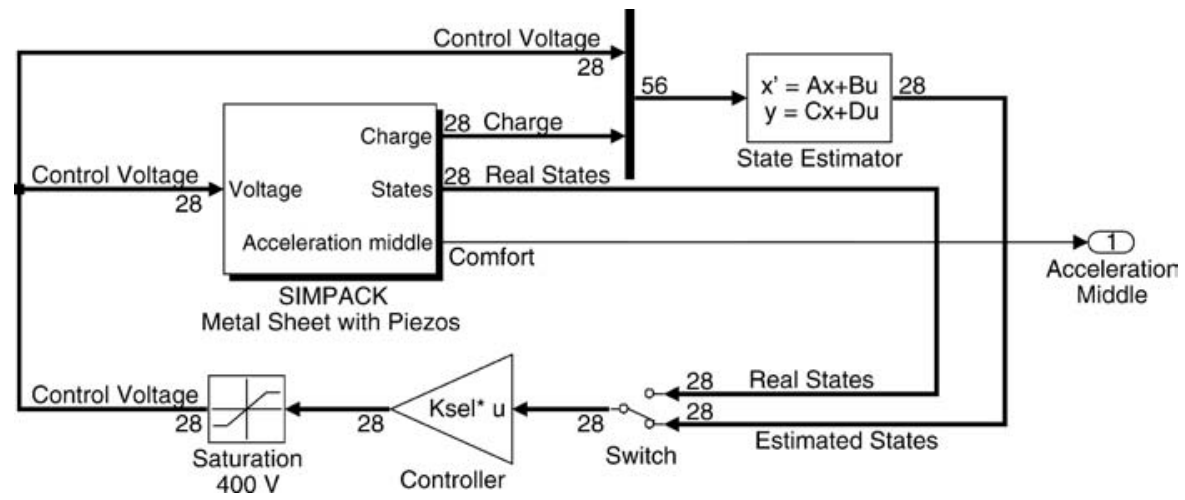

Figure 4. Simulink block diagram of the control loop.

control loop including the state estimator is presented in Figure 4. To drive the piezo-actuators within the linear range, the saturation block is proposed.

\subsection{SIMULATION RESULTS}

A comparison of the accelerations in the centre of the metal sheet is presented in Figure 5. The thin, light-grey line shows the response of the sheet without control. The only existing damping is the structural damping. The thick line represents the controlled systems from the Figure 4, which contains a state estimator and LQR feedback controller.

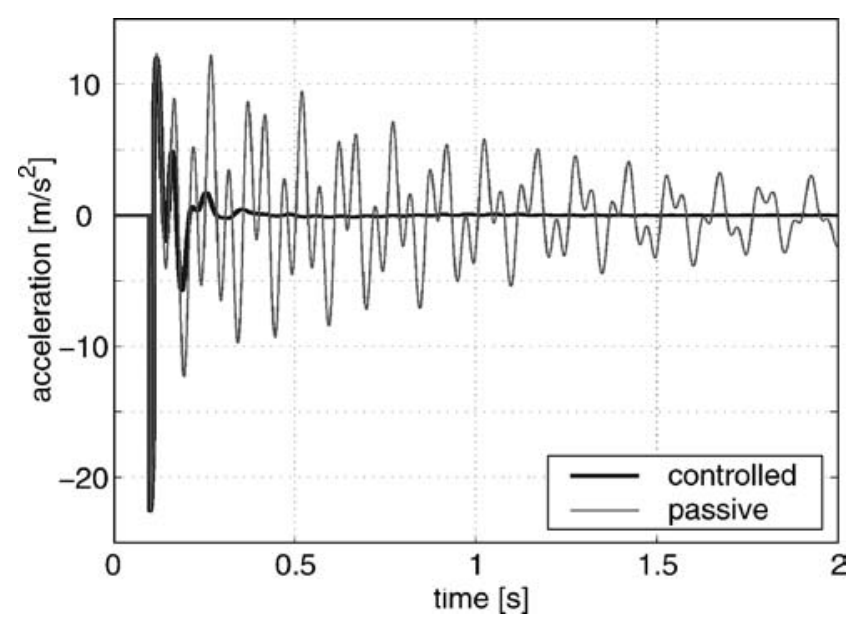

Figure 5. Acceleration in the centre of the metal sheet. 


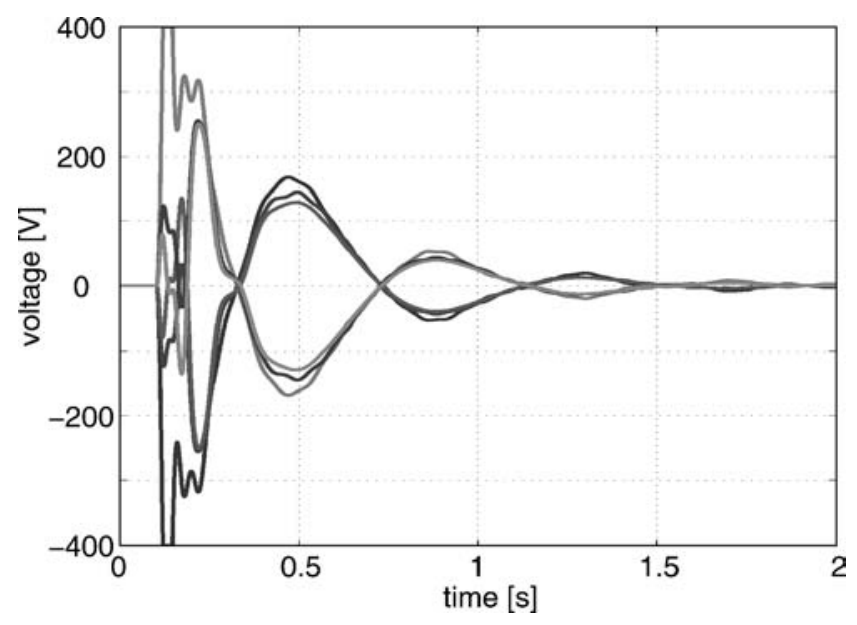

Figure 6. Voltage applied to the patches.

The corresponding voltages applied to the piezo-patches are presented in Figure 6 . The controller would remain within the linear range of the piezo-elements except for the maximum values during the force impact.

\section{Thermoelastic Applications}

In Section 3.1.2., an advanced modal reduction technique is proposed to represent coupled thermal and mechanical fields. The intention of the first case study, given later, is to justify this approach by example.

The second application in this section was chosen to give a comprehensible demonstration how normal stresses due to temperature distributions may influence the deflections of a body significantly.

\subsection{THERMAL DISC DEFORMATION}

A two-dimensional temperature and displacement field was simulated with the model illustrated in Figure 7. On one sector of the circular disc $(7 \mathrm{~mm}$ thick, outer radius $0.15 \mathrm{~m}$ ) a constant heat flux $q_{\mathrm{B}}=3000 \mathrm{~W} / \mathrm{m}^{2}$ was defined, the opposite sector was cooled by a fluid with a bulk temperature of $200 \mathrm{~K}$ below reference $\Theta_{0}$ $\left(h_{\mathrm{f}}=10 \mathrm{~W} /\left(\mathrm{m}^{2} \mathrm{~K}\right)\right)$. The displacements of the points of the inner circle (radius $0.075 \mathrm{~m}$ ) were set to zero. The material parameters are chosen as follows: $E=$ $2.1 \times 10^{11} \mathrm{~Pa}, v=0.3, \varrho=7800 \mathrm{~kg} / \mathrm{m}^{3}, \alpha=12 \times 10^{-6} \mathrm{~K}^{-1}, c=465 \mathrm{~J} /(\mathrm{kg} \mathrm{K})$, $\lambda=43 \mathrm{~W} /(\mathrm{m} \mathrm{K})$.

The light-grey areas in Figure 7 give an impression of the induced displacement field at $t_{e}=18,000 \mathrm{~s}$. The bars visualise the temperature distribution.

For verification, this scenario was evaluated in three set ups, a transient finite element simulation with 540 degrees of freedom and two different multibody models. 


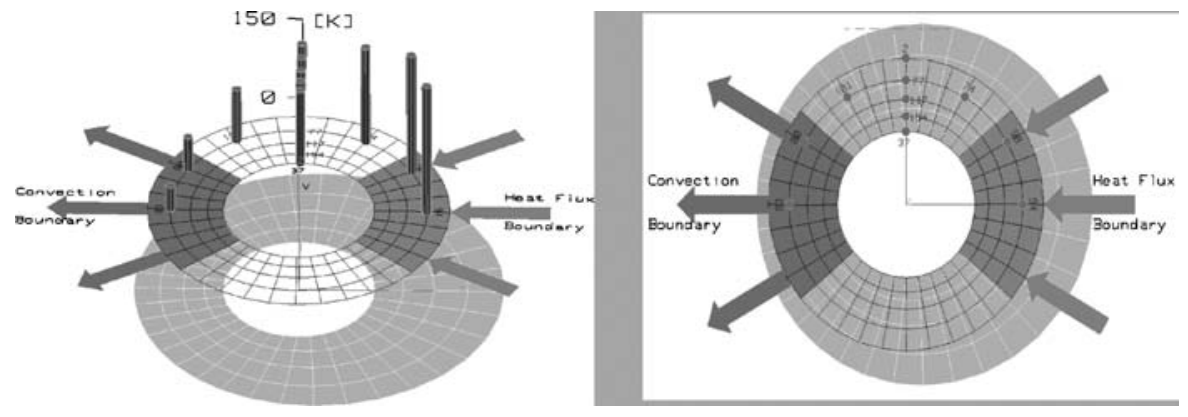

Figure 7. Definition, temperature and displacement results of the SIMPACK model Thermodisc.

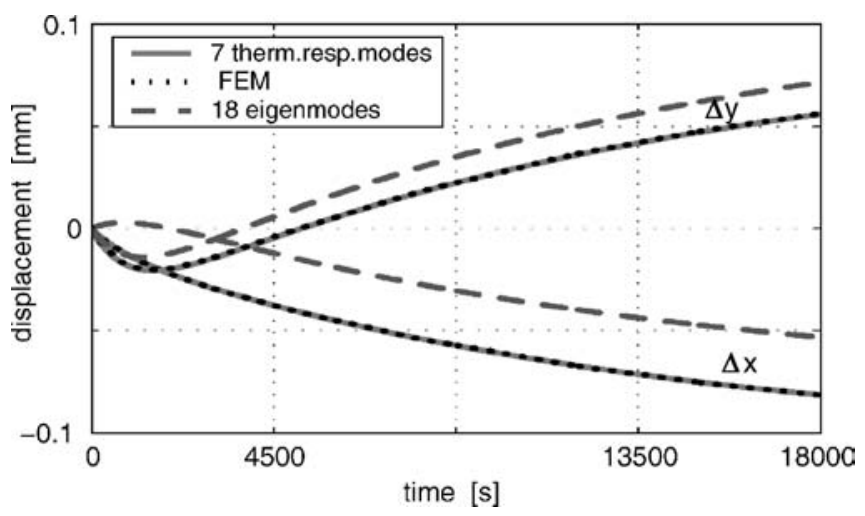

Figure 8. Transient displacements at node 101.

The first one consists of seven thermal modes to represent the temperature field and 18 preselected, plane mechanical eigenmodes. The second multibody model uses the same thermal eigenmodes, but for the displacement field the corresponding thermal response modes were selected.

Figure 9 distinguishes six thermal eigenmodes, whereas light areas represent lower temperatures. The corresponding thermal response modes are illustrated by the deformed mesh compared to the undeformed outer circle contour. The first mode, representing a spatially uniform temperature distribution, and the corresponding radial displacements are omitted.

Figure 8 compares the transient displacements at node 101. The finite element simulation and the multibody simulation with seven thermal response modes yield identical results, but the displacements caused by inhomogeneous temperature fields could not be represented by 18 purely mechanical eigenmodes. 

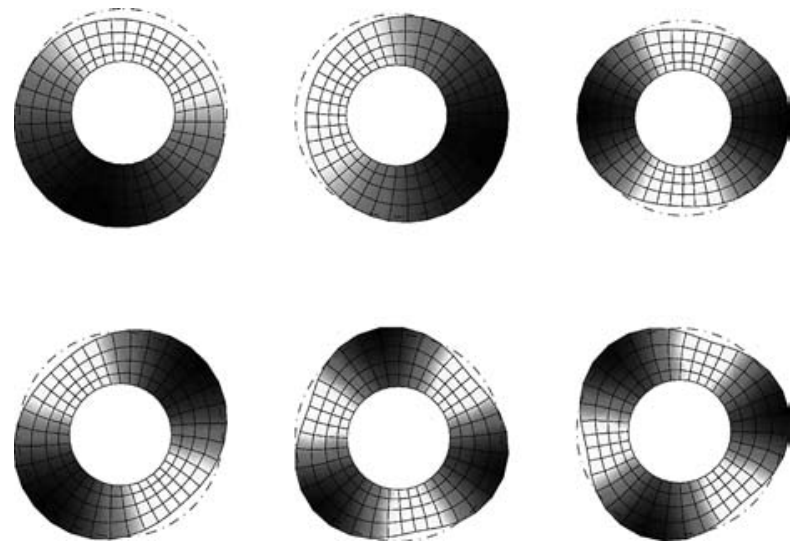

Figure 9. Thermal modes and corresponding thermal response modes.

\subsection{THERMAL BUCKLING OF A BEAM}

To demonstrate the mechanism of thermal buckling, an axially restrained beam, length $l=1 \mathrm{~m}$, was modelled in SIMPACK. The model considers the first bending eigenmode at $63 \mathrm{~Hz}$ and one linear axial expansion mode supposed to reflect the displacements caused by a uniform temperature distribution. Concerning its thermal properties, the beam was modelled as block capacity specified by one discrete temperature. It is assumed that the deformations remain completely within the realm of elasticity and small deflection theory.

The simulation scenario consists of a temperature rising linear in time. For illustration purposes, the beam was dynamically excited by a small lateral force, acting at the middle of the beam. In Figure 10 the response of the beam is represented

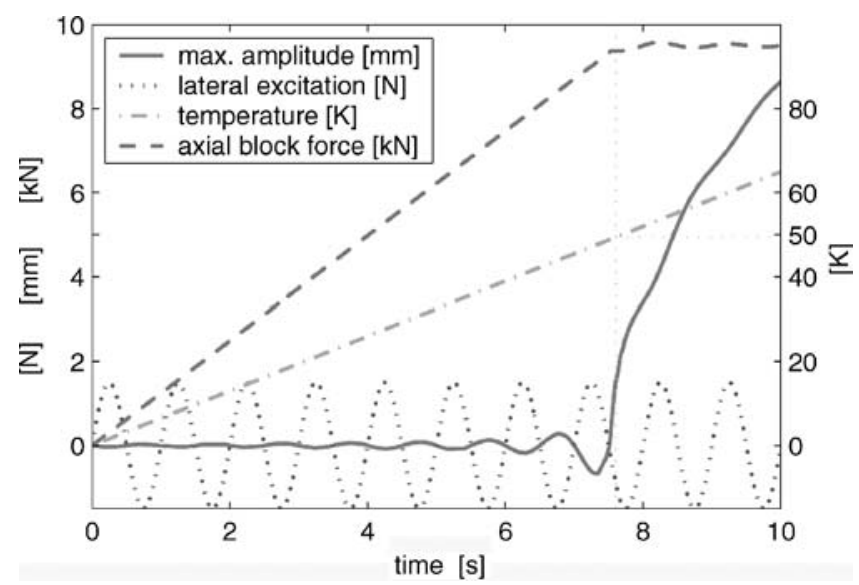

Figure 10. Simulation results of the SIMPACK model Thermobeam. 
by the amplitude at the beam's mid-point. As long as the absolute temperature is close to the reference temperature the reaction of the beam to the force excitation remains small. When the axial thrust force caused by the blocked thermal expansion reaches the critical value, the beam buckles into a new state of equilibrium. Later on the block force remains constant except for a small excitation response.

For verification, the differential equations governing the bending and axial deformation $w$ and $u$ are derived analytically on the basis of the work functions $T$, the kinetic energy, and $V$, the potential energy, specifying an axially unrestrained Rayleigh beam with additional thermal expansion (cf. [26, Section 13.3] and [27, Section II, C, 3]). The formulation includes an axial thrust force $F$ and its influence on $u$ and $w$. Symbol $A$ denotes the section area of the beam. $I$ is its geometrical moment of inertia. ( $)^{\prime}$ represents the partial derivative w.r.t. the co-ordinate $x$ :

$$
\begin{aligned}
T & =\frac{\varrho}{2} \int_{l}\left[A\left(\dot{u}^{2}+\dot{w}^{2}\right)+I \dot{w}^{\prime 2}\right] \mathrm{d} x \\
V & =\frac{1}{2} \int_{l}\left[E A u^{\prime 2}+E I w^{\prime \prime 2}+F\left(2 u^{\prime}-w^{\prime 2}\right)-(E A \alpha \vartheta) 2 u^{\prime}\right] \mathrm{d} x .
\end{aligned}
$$

Furthermore, a Ritz approach for both distributed variables is used and the magnitude of the load $F$ in (38) is determined by the condition that the displacement at the end of the beam is zero:

$$
w=\sin \frac{\pi x}{l} q(t), \quad u=\frac{x}{l} \bar{q}(t), \quad u(x=l)=\frac{1}{2} \int_{0}^{l} w^{\prime 2} \mathrm{~d} x .
$$

This approach enables the formulation of a single equation for the variable $q$, which describes the behaviour of the restrained beam including buckling:

$$
\begin{aligned}
\frac{\varrho}{2}\left[A l+\frac{I \pi^{2}}{l}+\frac{A \pi^{4}}{6 l} q^{2}\right] \ddot{q}+ & {\left[\frac{\varrho A \pi^{4}}{12 l} q\right] \dot{q}^{2}+} \\
& +\frac{E}{2 l}\left[\frac{I \pi^{4}}{l^{2}}-A \pi^{2} \alpha \vartheta+\frac{A \pi^{4}}{4 l^{2}} q^{2}\right] q=0
\end{aligned}
$$

Figure 11 presents some properties of the analytical system as function of the imposed temperature. The parameters are identical to those used for the SIMPACK simulation: $l=1 \mathrm{~m}, A=7.6 \times 10^{-5} \mathrm{~m}^{2}, I=4.585 \times 10^{-9} \mathrm{~m}^{4}, E=2.1 \times 10^{11} \mathrm{~Pa}$, $\varrho=7850 \mathrm{~kg} / \mathrm{m}^{3}, \alpha=12 \times 10^{-6} \mathrm{~K}^{-1}$.

The bifurcation point at $50 \mathrm{~K}$ in Figure 11 is marked with circles. Within the considered temperature range, the bending amplitude reaches values below $9 \mathrm{~mm}$, which is small enough to justify geometrical linearisation. Figure 11 corresponds well with the results of the SIMPACK model in Figure 10. 


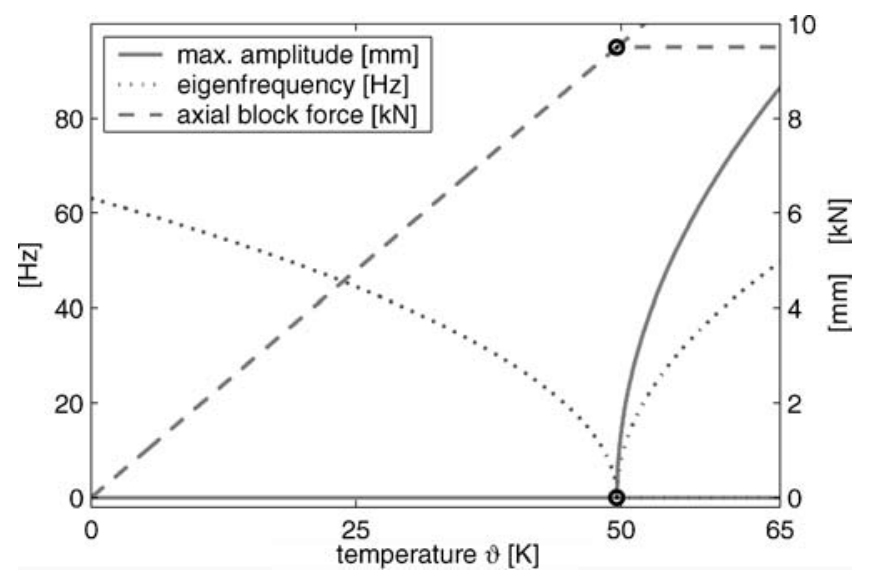

Figure 11. Properties of the analytical buckling model in state of equilibrium.

\section{Conclusions and Open Problems}

A general concept for the simulation of multiphysical phenomena in a multibody dynamics environment has been presented. Its feasibility has been demonstrated on examples. The proposed concept, the modal multifield approach, enables a lowdimensional description of bodies with multiple distributed properties. The capabilities of multibody dynamics, in particular its numerical efficiency, can now be used to design, optimise and control systems with adaptive devices or thermoelastic behaviour.

The specific challenge of smart structure evaluation may be found in the fact that the physical description is only one part of the actual task. At least the same importance may be attached to the design optimisation problem and the set up of appropriate control concepts. It should also be noted that the presented theory is based on linear piezoelectricity. Therefore, the present paper does not account for piezo-patches which are driven in large signal mode and expose saturation and hysteresis effects.

Regarding thermoelasticity, additional investigations are necessary on the limits of the linear temperature field description and the influence of the Gough-Joule effect, particularly concerning high-frequency excitations. However, in most industrial applications, transient temperature field simulation is not requested anyway because of very long simulation time periods. These applications will be handled favourably under quasi-stationary thermal conditions, provided that reliable strategies are available to define these conditions.

\section{Acknowledgments}

The authors would like to acknowledge the collaboration and the kind support provided by Dr. Stefan Dietz, Dr. Lutz Mauer and Dr. Wolfgang Rulka from INTEC 
GmbH (Germany). Furthermore, the authors appreciate the co-operation of the Adaptronics Section, particularly Dr. Michael Rose and its former head Prof. Dr. Delf Sachau, DLR Institute of Structural Mechanics in Braunschweig (Germany).

\section{References}

1. Arnold, M., Carrarini, A., Heckmann, A. and Hippmann, G., 'Modular dynamical simulation of mechatronic and coupled systems' in Proceedings of WCCM V, Fifth World Congress on Computational Mechanics, Mang, H.A., Rammerstorfer, F.G. and Eberhardsteiner, J. (eds.), Vienna, 2002, http://wccm.tuwien.ac.at.

2. Heckmann, A., Mehrkörpersimulation des thermoelastischen Modells einer Werkzeugmaschine, Internal Report IB 532-2003-03, DLR German Aerospace Center, Institute of Aeroelasticity, Vehicle System Dynamics, Oberpfaffenhofen, 2003.

3. Tichý, J. and Gautschi, G., Piezoelektrische Messtechnik. Springer, Berlin, 1980.

4. Nowinski, J.L.H., Theory of Thermoelasticity with Applications. Sijthof \& Noordhoff International Publishers B.V., Alphen aan den Rijn, The Netherlands, 1978.

5. Zienkiewicz, O.C. and Taylor, R.L., The Finite Element Method, 5th edn. Butterworth Heinemann, Oxford, 2000.

6. Parkus, H., Variational Principles in Thermo- and Magneto-Elasticity. Springer, Wien, 1970.

7. Nowacki, W., 'Some general theorems of thermopiezoelectricity', Journal of Thermal Stresses 1, 1978, 171-182.

8. Lanczos, C., The Variational Principles of Mechanics, 4th edn. Dover, New York, 1970.

9. Schwertassek, R. and Wallrapp, O., Dynamik flexibler Mehrkörpersysteme. Vieweg Verlag, Braunschweig, 1999.

10. Shabana, A.A., Dynamics of Multibody Systems, 2nd edn. Cambridge University Press, Cambridge, 1998.

11. Preumont, A., Vibration Control of Active Structures, 2nd edn. Academic Publishers, Dordrecht, 2002.

12. Rose, M. and Sachau, D., 'Multibody simulation of mechanism with distributed actuators on lightweight components', in Proceedings of the SPIE's 8th Annual International Symposium on Smart Structures and Materials, Newport Beach, 2001.

13. Lienhard IV, J.H. and Lienhard V, J.H., A Heat Transfer Textbook. Phlogiston Press, Cambridge, MA, 2001.

14. Schweizer, B. and Wauer, J., 'Atomistic explanation of the Gough-Joule-effect', The European Physical Journal B 23, 2001, 383-390.

15. Rulka, W., 'Effiziente Simulation der Dynamik mechatronischer Systeme für industrielle Anwendungen', Ph.D. Thesis, Vienna University of Technology, 1998.

16. Schiehlen, W., 'Multibody dynamics: Roots and perspectives', Multibody System Dynamics 1, 1997, 149-188.

17. Piefort, V., 'Finite element modelling of piezoelectric active structures', Ph.D. Thesis, Université Libre de Bruxelles, 2001.

18. Heckmann, A. and Vaculín, O., 'Multibody simulation of actively controlled carbody flexibility', in Proceedings of the International Conference on Noise and Vibration Engineering (ISMA 2002), Sas, P. and van Hal, B. (eds.), Leuven, Belgium, 2002, 1123-1132.

19. Dietz, S., Vibration and Fatigue Analysis of Vehicle Systems Using Component Modes. VDIVerlag, Düsseldorf, 1999.

20. Simeon, B., Numerische Simulation gekoppelter Systeme von partiellen und differentialalgebraischen Gleichungen in der Mehrkörperdynamik. Fortschritt-Berichte VDI Reihe 20, Nr. 325, VDI-Verlag, Düsseldorf, 2000. 
21. Brenan, K.E., Campbell, S.L. and Petzold, L.R., Numerical Solution of Initial-Value Problems in Differential-Algebraic Equations, 2nd edn. SIAM, Philadelphia, 1996.

22. Veitl, A. and Arnold, M., 'Coupled simulation of multibody systems and elastic structures', in Advances in Computational Multibody Dynamics, Ambrósio, J.A.C. and Schiehlen, W.O. (eds.), IDMEC/IST Lisbon, Portugal, 1999, 635-644.

23. Dietz, S., Hippmann, G. and Schupp, G., 'Interaction of vehicles and flexible tracks by cosimulation of multibody vehicle systems and finite element track models', in 17th Symposium Dynamics of Vehicles on Roads and Tracks IAVSD 2001, August 20-24, Copenhagen, 2001.

24. Kortüm, W., Schiehlen, W. and Arnold, M., 'Software tools: From multibody system analysis to vehicle system dynamics', in Proceedings of the International Congress on Theoretical and Applied Mechanics, ICTAM 2000, Chicago, USA, 2000, 225-238.

25. Vaculín, O., Krüger, W.-R. and Spieck, M., 'Coupling of multibody and control simulation tools for the design of mechatronic systems', in ASME 2001 Design Engineering Technical Conferences, DETC2001/VIB-21323, Pittsburgh, 2001.

26. Boley, B.A. and Weiner, J.H., Theory of Thermal Stresses. Dover, Mineola, New York, 1997.

27. Pflüger, A., Stabilitätsprobleme der Elastostatik. Springer, Berlin, 1975. 